Published in "Physica A: Statistical Mechanics and its Applications 494: 403-409, 2018"

which should be cited to refer to this work.

\title{
Uncovering the popularity mechanisms for Facebook applications
}

\author{
Sheng-Nan Li ${ }^{\text {a }}$, Qiang Guo ${ }^{\text {a }}$, Kai Yang ${ }^{\text {a }}$, Jian-Guo Liu ${ }^{\text {b,* }}$, Yi-Cheng Zhang ${ }^{\mathrm{c}}$ \\ ${ }^{a}$ Research Center of Complex Systems Science, University of Shanghai for Science and Technology, Shanghai 200093, PR China \\ b Data Science and Cloud Service Research Centre, Shanghai University of Finance and Economics, Shanghai 200433, PR China \\ ' Department of Physics, University of Fribourg, Fribourg $\mathrm{CH}-1700$, Switzerland
}

\section{H I G H L I G H T S}

- For different kinds of Facebook Apps and found that the recent and cumulative popularity play important roles.

- We present a model to regenerate the growth of popularity for different App groups.

- The recent popularity plays more important role in regenerating the popularity dynamics for more popular Apps.

- The cumulative popularity plays more important role for unpopular Apps.

\begin{abstract}
Understanding the popularity dynamics of online application(App) is significant for the online social systems. In this paper, by dividing the Facebook Apps into different groups in terms of their popularities, we empirically investigate the popularity dynamics for different kinds of Facebook Apps. Then, taking into account the influence of cumulative and recent popularities on the user choice, we present a model to regenerate the growth of popularity for different App groups. The experimental results of 917 Facebook Apps show that as the popularities of Facebook Apps increase, the recent popularity plays more important role. Specifically, the recent popularity plays more important role in regenerating the popularity dynamics for more popular Apps, and the cumulative popularity plays more important role for unpopular Apps. We also conduct temporal analysis on the growth characteristic of individual App by comparing the increment at each time with the average of historical records. The results show that the growth of more popular App tends to fluctuate more greatly. Our work may shed some lights for deeply understanding the popularity mechanism for online applications.
\end{abstract}

\section{Introduction}

Popularity dynamic plays an important role in online social systems [1]. The mechanisms for online object popularity have been widely studied including movies [2], musics [3], news and other online social collective behaviors [4,5]. Many factors affect the object popularity, such as the internal quality [6], ranking list [7], brand effect [8], recommendation system [9,10], social communication [11,12], and so on [13-15].

\footnotetext{
* Corresponding author.

E-mail address: liujg004@ustc.edu.cn (J. Liu).
} 


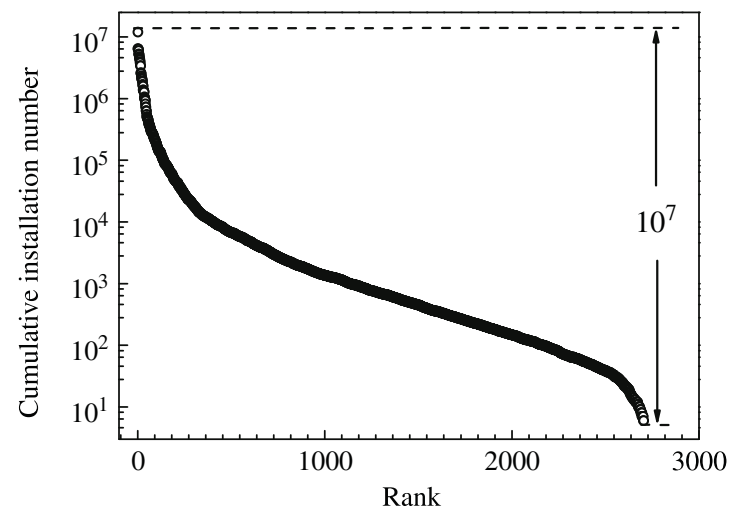

Fig. 1. The number of cumulative installation for 2705 Facebook Apps before August 14, 2007. The vertical axis shows Apps' final cumulative installation number, and horizontal axis reflects the corresponding rank. The difference between the smallest and the largest number of cumulative installation is over ten millions, which indicates the gap in popularities among online Apps.

The preferential selection is regarded as a classic factor on object popularity, which leads to a "rich-get-richer" phenomenon that objects with more cumulative selections also tend to attract more attentions [16,17]. Borghol et al. [18] empirically measured the popularity of videos and found that preferential selection could be used to interpret the video popularity evolution. Szabo et al. [19] found that the long time popularity of online content could be predicted by the early user accesses. Shen et al. [20] introduced the reinforcement Poisson mechanism to model and predict the popularity dynamics. Comparing with the rich-get-richer phenomenon, Bentley et al. [21] introduced a "memory" parameter defined as the number of previous steps which affects an individual's decision. Gleeson et al. [22] investigated the popularity dynamic of the Facebook Apps and found that recent popularity plays more important role.

However, it should be noticed that the popularities of most empirical systems are heterogeneous [23]. Medo et al. [24] found that the citation network exhibits heterogeneous fitness values. For the object popularity of the user-object networks, Liu et al. [25] found that the online user interests could be divided as common interests and specific interests. Furthermore, $\mathrm{Ni}$ and Wang et al. $[26,27]$ found that small-degree users tend to select popular movies, while large-degree users prefer to select the unpopular ones. The heterogeneous physics of the object popularity plays an important role for the online social systems evolution [28,29]. As shown in Fig. 1, for the popularity of Facebook Apps, the smallest cumulative installation number is only six, while the largest installation number is over ten millions.

In this paper, we investigate the roles of the cumulative and recent popularities in the popularity mechanisms for Facebook Apps. By dividing the Facebook Apps into different groups in terms of their popularities, we empirically investigate the popularity dynamics of Apps with different popularities and find that the growth rate of more unpopular Apps fluctuates more randomly in the initial period, and the growth rate of all App groups finally stabilizes around 1 . Then, we present a model to regenerate the popularity dynamics of the empirical Facebook Apps, and find that as the Apps popularities increase, the recent popularity plays more important role. Finally, we conduct temporal analysis on the growth characteristic of each App by comparing the increment at each time step with the average installation time of historical records, which show that the growth of online Apps is fluctuant and the growth of more popular App fluctuates more greatly.

\section{Empirical analysis}

In this section, we analyze the popularity dynamics for different kinds of Apps by dividing Facebook Apps in terms of their popularity [30]. Firstly, we introduce the definitions to measure Apps' popularities [22]. To measure the change in number of cumulative installation for App $i$ at time $t$, the increment $f_{i}(t)$ can be denoted by

$$
f_{i}(t)=n_{i}(t)-n_{i}(t-1),
$$

where $n_{i}(t)$ is the total number of users who have installed App $i$ by time $t$.

To compare the popularities of different Apps when they are the same age (i.e., the same number of time steps after they were launched), the age-shifted increment $\widetilde{f}_{i}(a)$ was introduced to measure the change in cumulative installation number for App $i$ at age $a$, which is defined as $\widetilde{f}_{i}(a)=\widetilde{n}_{i}(a)-\widetilde{n}_{i}(a-1)$, where $\widetilde{n}_{i}(a)=n_{i}\left(t_{i}+a\right)$ is the cumulative installation number of App $i$ during $a$ time steps after its launch time $t_{i}$. In order to present the popularity dynamics for a group of Apps, the mean scaled age-shifted growth rate $r(a)$ can be defined as

$$
r(a)=\left\langle\frac{\tilde{f}_{i}(a)}{u_{i}}\right\rangle_{z}
$$


Table 1

The basic information about each App group, where $N_{Z}$ is the number of Apps containing in each group $Z$.

\begin{tabular}{llr}
\hline$Z$ & Range of $\log \left(u_{i}\right)$ & $N_{Z}$ \\
\hline$Z_{1}$ & {$[-3,-1)$} & 94 \\
$Z_{2}$ & {$[-1,0)$} & 381 \\
$Z_{3}$ & {$[0,1)$} & 296 \\
$Z_{4}$ & {$[1,2)$} & 112 \\
$Z_{5}$ & {$[2,3)$} & 26 \\
$Z_{6}$ & {$[3,4)$} & 8 \\
\hline
\end{tabular}

where $\langle\cdot\rangle_{Z}$ denotes an ensemble average over all Apps in a group $Z$, and $u_{i}$ is the temporal average increment of App $i$ over its first $t_{\text {half }}$ observations (i.e., more than one half of the data collection window), which could be written as

$$
u_{i}=\frac{\sum_{a=1}^{t_{\text {half }}} \tilde{f}_{i}(a)}{t_{\text {half }}} .
$$

By weighting Apps over the same number of observations, the temporal average increment $u_{i}$ measures the average popularity of each App comparably among all.

\subsection{Description of the dataset}

The original dataset includes the number of cumulative installations for every Facebook App in each hour from June 25, 2007 to August 14, 2007, spanning $t_{\max }=1209 \mathrm{~h}(n=2705$ Apps total available during this period $)$ [31]. When $t=0$, there are already 980 Apps in the system, whose launch times are unknown. In this paper, we only take into account the Apps which are launched after the data collection and have at least $t_{\text {half }} \equiv 650 \mathrm{~h}$ observations. Denoting the launch time of App $i$ by $t_{i}$, the selected Apps satisfy the following conditions: $t_{i}>0$ and $t_{i}<t_{\max }-t_{\text {half }}=559$. Finally, the amount of Apps mentioned in our following study is $N_{\text {total }}=917$.

\subsection{Empirical results}

Considering that the distribution of Apps' average popularity value $u_{i}$ is heavy-tailed, we divide the 917 Apps into 6 groups by calculating the corresponding logarithm of their $u_{i}$ values. Specifically, the $\log \left(u_{i}\right)$ values are set as $[-3,-1),[-1,0),[0$, $1),[1,2),[2,3)$ and $[3,4)$, respectively. The basic information of each group is shown in Table 1 . Apps in the first three groups are unpopular Apps, and Apps in another three groups are popular Apps.

Then we analyze the popularity dynamics for different App groups, which are reflected by the mean scaled age-shifted growth rate $r(a)$. The empirical analysis results for six groups of popular and unpopular Apps are shown in Fig. 2. In Fig. 2(A)(C), the growth rates of the unpopular Apps fluctuate randomly with large values in initial period. Specifically, the maximum $r(a)$ for more unpopular Apps is larger, which are 19.03, 7.05 and 3.89 respectively as shown in Fig. 2(A)-(C). In Fig. 2(D)-(F), the growth rate of the popular Apps fluctuate with 24-h period, which gradually increase in initial period. In addition, the growth rates of all App groups finally stabilize around 1.

Actually, the difference in popularity dynamics can be explained with lots of factors. Namely, one can imagine that newly launched Apps are initially installed by friends of the authors of the Apps due to social support [32]. Imagine that each App receives the same amount of social support. Then, the quality Apps will start to aggregate installations and will become popular eventually. However, the unpopular Apps (maybe with the poor quality or inconvenient function) are only installed at initial launch and soon after that are forgotten by public, which brings about the relatively large values in initial period of the growth rate $r(a)$. The investigation simply dealing with Apps all together may ignore the different popularity dynamics and interfere with the accuracy of experimental results.

The popularity dynamics are different among Apps groups, which could indicate that the popularity mechanisms for different kinds of Apps are heterogeneous. Therefore, we construct model to investigate the popularity mechanisms of online Apps with different popularities.

\section{Regenerative model construction}

In this section, we present a model incorporating the cumulative and recent popularities to regenerate the Facebook Apps popularity dynamics which are presented by the mean scaled age-shifted growth rate $r(a)$. The cumulative popularity [16] and recent popularity [22] could affect the probability of individual choice. The cumulative popularity in our model is corresponding with the "rich-get-richer" phenomenon that Apps with more previous installations also tend to attract more adoptions, and the recent popularity is defined as the number of installations grown recently. 



Fig. 2. The mean scaled age-shifted growth rate $r(a)$ for different kinds of Apps, which are divided into six groups according to the logarithm of their average popularity $u_{i}$, and Apps' $\log \left(u_{i}\right)$ values are set as $[-3,-1),[-1,0),[0,1),[1,2),[2,3)$ and $[3,4)$, respectively. The horizontal axis denotes the number of hours after Apps' launch. The results in the subplots (A)-(C) show that the growth rate of more unpopular Apps fluctuate more randomly with larger values in initial period, while the results in the subplots (D)-(F) show that the growth rate of popular Apps fluctuate with steady 24-h period.

We redistribute the total increment $F_{Z}(a)$ of Apps in group $Z$ at each age $a$. The $F_{Z}(a)$ values are taken from the empirical data by following equation:

$$
F_{Z}(a)=\sum_{i \in Z} \widetilde{f}_{i}(a)
$$

where $Z=\left\{Z_{1}, Z_{2}, \ldots, Z_{6}\right\}$ contains six App groups.

For each group $Z_{j}(j=1,2, \ldots, 6)$, the total increment $F_{Z_{j}}(a)$ at each age $a$ is redistributed to each App $i$ that including in group $Z_{j}$ with probability $p_{i}(a)$, which is set as a weighted sum,

$$
p_{i}(a)=\gamma p_{i}^{c}(a)+(1-\gamma) p_{i}^{r}(a)
$$

where $p_{i}^{c}(a)$ is the "cumulative-popularity rule" that users choose App $i$ at age $a$ with a probability proportional to its cumulative popularity $\widetilde{n}_{i}(a-1)$, yielding

$$
p_{i}^{c}(a)=\frac{\tilde{n}_{i}(a-1)}{\sum_{i \in Z_{j}} \widetilde{n}_{i}(a-1)},(j=1,2, \ldots, 6),
$$

and $p_{i}^{r}(a)$ is the "recent-popularity rule" that users choose App $i$ at age $a$ with a probability proportional to its recent popularity, which is described as

$$
p_{i}^{r}(a)=\frac{\sum_{\tau=1}^{a-1} e^{-(a-\tau)} \widetilde{f}_{i}(\tau)}{\sum_{i \in Z_{j}} \sum_{\tau=1}^{a-1} e^{-(a-\tau)} \widetilde{f}_{i}(\tau)},(j=1,2, \ldots, 6),
$$

where $e^{-(a-\tau)}$ is an exponential "memory" function which assigns weight to the age-shifted increment $\widetilde{f}_{i}$ from $\tau$ hours ago $[21,33]$.

In the process of regenerating at each time step, the $\tilde{n}_{i}(a)$ in Eq.(6) and $\tilde{f}_{i}(\tau)$ in Eq.(7) are obtained from the real data to determine the probability $p_{i}(a)$. The installation probability $p_{i}(a)$ interpolates between the extremes of $\gamma=0$ (recent popularity) and $\gamma=1$ (cumulative popularity). In each group $Z_{j}$, after distributing each total increment $F_{Z_{j}}(a)$, we regenerate the age-shifted increment $\widetilde{f}_{i}(a)$ of each App $i$ from $a=2$ to $a=650$, and the App's initial age-shifted increment $\tilde{f}_{i}(1)$ is extracted from the empirical data. 


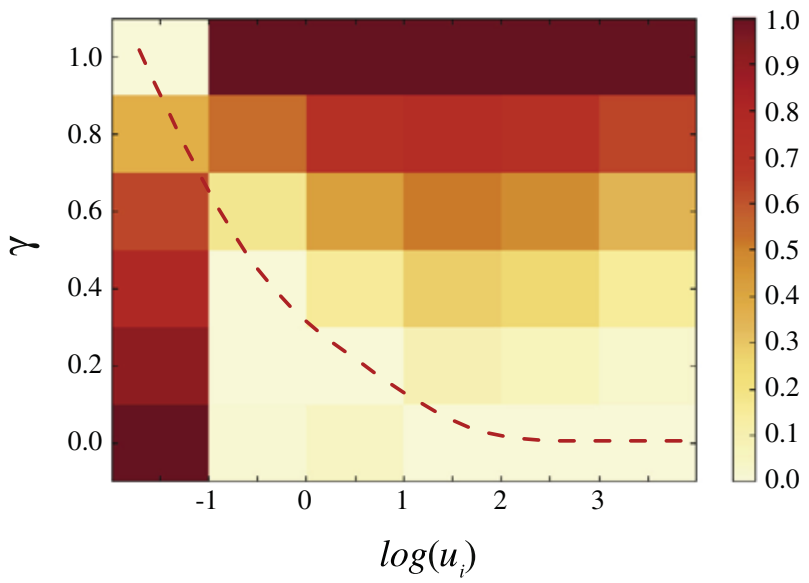

Fig. 3. The normalized difference $E^{Z_{j}}{ }^{*}(\gamma)$ under different weight coefficients $\gamma$ for six App groups dividing in terms of the logarithm of Apps popularities $\log \left(u_{i}\right)$, where the dashed line reflects the optimal parameter $\gamma^{*}$ of the regenerative model for different App groups. From the experimental results, one can find that as the Apps' popularities increase, the optimal parameter $\gamma^{*}$ would decrease from 1 to 0 , suggesting that the recent popularity plays more important role for popular Apps.

\subsection{Measurement}

For each App group $Z_{j}$, to investigate the performance of cumulative and recent popularities for App's popularity, we measure the difference $E^{Z_{j}}(\gamma)$ between the regenerated $r(a)$ under different weight coefficients $\gamma$ denoted by $r_{r e g}^{\gamma}$ and the empirical data denoted by $r_{\text {data }}$ during first $t_{\text {half }}=650$ time steps, which could be written as

$$
E^{Z_{j}}(\gamma)=\sqrt{\sum_{a=1}^{t_{\text {half }}}\left(r_{\text {reg }}^{\gamma}(a)-r_{\text {data }}(a)\right)^{2}}
$$

where $j=1,2, \ldots, 6$, and $\gamma=0,0.2, \ldots, 1$.

To compare the differences $E^{Z_{j}}(\gamma)$ in each App group, we normalize the $E^{Z_{j}}$ values under different weight coefficients $\gamma$ of each group, which is defined as $E^{Z_{j}^{*}}(\gamma)$,

$$
E^{Z_{j}}(\gamma)=\frac{E^{Z_{j}}(\gamma)-\min E^{Z_{j}}}{\max E^{Z_{j}}-\min E^{Z_{j}}}
$$

where $\min E^{Z_{j}}$ and $\max E^{Z_{j}}$ are the minimum and maximum of $E^{Z_{j}}(\gamma)$ values under different weight coefficients $\gamma$ in the group $Z_{j}$. The normalized difference $E^{Z_{j}{ }^{*}}$ for each group has a value between 0 and 1 , where $E^{Z_{j}}{ }^{*}(\gamma)=0$ means that the regenerative performance fits the most closely with the empirical data, thus the $\gamma$ that leads to $E^{Z_{j}}(\gamma)=0$ is the optimal parameters $\gamma^{*}$ of the regenerative model for corresponding App group $Z_{j}$.

\subsection{Experimental results}

The normalized difference $E^{Z_{j}}{ }^{*}(\gamma)$ and the optimal parameters $\gamma^{*}$ of the regenerative model of each App group are shown in Fig. 3, from which one can find that as the popularities of Apps increase, the recent popularity plays more important role. Specifically, for regenerating in groups of popular Apps, the optimal parameter $\gamma^{*}$ changes to 0 , while the optimal parameter $\gamma^{*}$ equals to 1 for the most unpopular Apps. That is to say the users' choice among popular Apps is more likely to be affected by the Apps' recent popularities, while choosing among unpopular Apps is affected more by Apps' cumulative popularities.

After investigating the popularity mechanisms for different App groups, we focus on investigate the popularity for individual App. Considering the fact that Apps' popularities are result of attracting more and more user attentions over time [34,35], we continue to conduct temporal analysis of growth characteristic for each App.

\section{Growth characteristic of individual App}

In this section, we conduct the temporal analysis of growth characteristic for every App, by comparing the installation increment in each period with average-level of the historical records. At each age $a$ in the growth duration of Facebook App $i$, we define the growth fluctuation $S_{i}(a)$ as follow,

$$
S_{i}(a)=\widetilde{f}_{i}(a)-\frac{\sum_{a^{\prime}=1}^{a-1} \widetilde{f}_{i}\left(a^{\prime}\right)}{a-1}
$$




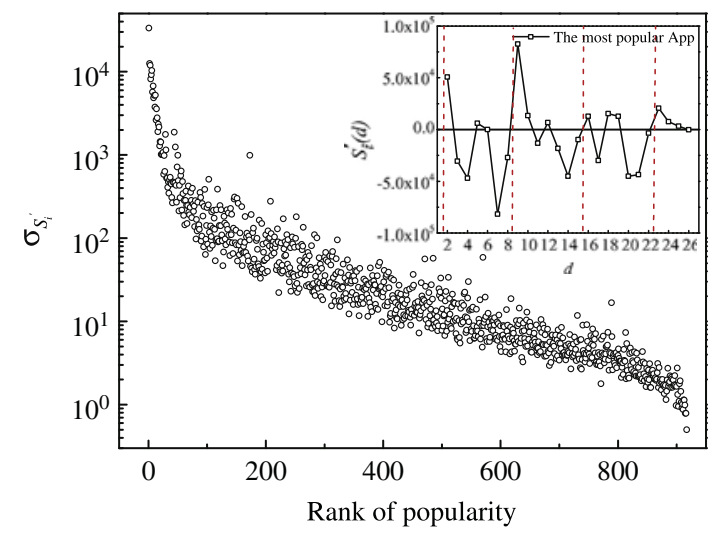

Fig. 4. The growth fluctuation of 917 Apps, where $\sigma_{S_{i}^{\prime}}$ is the standard deviation of 27 days extended growth fluctuation sequence $S_{i}^{\prime}(d)$ which is calculated by comparing the increment of App $i$ at time $d, \widetilde{f}_{i}^{\prime}(d)$, with the average-level of its historical records, $\sum_{d^{\prime}=1}^{d-1} \widetilde{f}_{i}^{\prime}\left(d^{\prime}\right) / d-1$, and $d$ is the number of App's existing days. The correlation between growth fluctuation of 27 days $\sigma_{S_{i}^{\prime}}$ and App's popularity $u_{i}$ indicates that as the popularities of Apps increase, the growth of Apps fluctuate more greatly. The subplot shows the extended growth fluctuation $S_{i}^{\prime}(d)$ of the most popular App, from which one can find that its growth is fluctuant and exists 7-days periods.

where $S_{i}(a)=0$ means that the increment of installations for App $i$ at age $a$ is at the same level with the average of all previous period, and $S_{i}(a)>0$ means that the App $i$ grows faster at age $a$ than previous average installation, and vice versa.

To remove the 24-h periodicity of function $S_{i}(a)$, we extend the period from an hour to a day represented by variable $d$, and the extended growth fluctuation is expressed as

$$
S_{i}^{\prime}(d)=\widetilde{f}_{i}^{\prime}(d)-\frac{\sum_{d^{\prime}=1}^{d-1} \widetilde{f}_{i}^{\prime}\left(d^{\prime}\right)}{d-1}
$$

where $\widetilde{f}_{i}^{\prime}(d)$ is the sum of periodical age-shifted increment $\widetilde{f}_{i}(a)$ for the $d$ th $24 \mathrm{~h}$. Limited in the shortest observation duration of the 917 Apps which is $650 \mathrm{~h}$, our temporal analysis covers the first 27 days after the App's launch (i.e., $d \in[2,27]$ ).

In order to measure the extended growth fluctuation $S_{i}^{\prime}(d)$ in total 27 days for each App $i$, we calculate the standard deviation $\sigma_{S_{i}^{\prime}}$ of the extended growth fluctuation $S_{i}^{\prime}(d)$ sequence. A larger $\sigma_{S_{i}^{\prime}}$ value reflects a more greatly fluctuation during the growth of App $i$.

The result of the standard deviation $\sigma_{S_{i}^{\prime}}$ for all the 917 Apps are shown in Fig. 4, which indicates that as the popularities of Apps increase, the growth of Apps tend to fluctuate more greatly. Specifically, the standard deviation $\sigma_{S_{i}^{\prime}}$ of the extended growth fluctuation $S_{i}^{\prime}(d)$ for the most popular App is more than $3 \times 10^{4}$, and that for the App with the least popularity $u_{i}$ decreases to below 0.5 . Furthermore, we take the extended growth fluctuation $S_{i}^{\prime}(d)$ sequence of the most popular App for example, which shows that its growth is fluctuant and exists 7-days periods, in each of which the $S_{i}^{\prime}(d)$ values are larger at beginning and tend to reduce later. The characteristic of growth fluctuation and the larger standard deviation values of the more popular Apps indicate that the development to the popular is not growing continuously, and the great fluctuation is more likely to bring great growth.

\section{Conclusion and discussions}

In this paper, taking into account the physics of popularity dynamics for online social systems [23], we investigate the popularity mechanisms for different groups of Facebook Apps divided in terms of their average popularity. Firstly, we empirically analyze the popularity dynamics of different kinds of Apps, where the growth rates of popular Apps fluctuate with a steady 24-h cycle and the growth rate of more unpopular Apps fluctuates more randomly with a larger value at initial period. Then, we propose a model by combining cumulative and recent popularities to regenerate the empirical growth rates, and to investigate the optimal popularity mechanisms for different App groups. The experimental results show that as the Apps' popularities increase, the recent popularity plays more important role in attracting more user choice. Specifically, the cumulative popularity plays more important role for the unpopular Apps, and the recent popularity plays more important role for popular Apps. Finally, in order to investigate the growth characteristics of individual App, we temporally analyze the growth characteristics of each of the 917 Facebook Apps by comparing the increment at each time step with the average of historical records. The result shows that as the Apps' popularities increase, the Apps growth of attracting more installations fluctuate more greatly. Specifically, the standard deviations of 27 days growth fluctuation sequence of the 917 Apps increase with the rising of Apps' popularities.

We investigated the heterogeneous popularity mechanisms of online Apps [36], and found that neither the recent popularity nor cumulative popularity can guide the growth of all kinds of Apps, which could form some new insights in 
the popularity prediction. And our results also show that the users' choice of different types of online contents are not only affected by a simply factor, thus considering the different properties among different populations, and analyzing separately if possible are necessary for accurate prediction of the growth. However, on the one hand, we would enhance our model to regenerate the long-term popularity of online objects [37] and consider more popularity mechanisms from the perspective of social system in our future works, such as community [38-40], trust relationship, and friend relationship, and so on. On the other hand, the potential factors on Apps' becoming popular is still a meaningful field to improve the popularity of online objects. Nowadays, the smart phones become more and more popular, and people spend more and more time on playing phones for different kinds of purposes and preferences, thus uncovering the popularity dynamics for different kinds of Apps or different populations at recent time is also a meaningful question.

\section{Acknowledgments}

We acknowledge Jukka-Pekka Onnela and Felix Reed-Tsochas Research Group for providing us the Facebook Apps data. This work is partially supported by the National Natural Science Foundation of China (Grant Nos. 61773248, 61374177, 71371125).

\section{References}

[1] A. Vespignani, Predicting the behavior of techno-social systems, Science 325 (5939) (2009) 425-428.

[2] J.G. Liu, L. Hou, Y.L. Zhang, W.J. Song, X. Pan, Empirical analysis of the clustering coefficient in the user-object bipartite networks, Internat. J. Modern Phys. C 24 (08) (2013) 1350055.

[3] T. Zhou, Z. Kuscsik, J.G. Liu, M. Medo, J.R. Wakeling, Y.C. Zhang, Solving the apparent diversity-accuracy dilemma of recommender systems, Proc. Natl. Acad. Sci. USA 107 (10) (2010) 4511-4515.

[4] R.M. Bond, C.J. Fariss, J.J. Jones, A.D.I. Kramer, C. Marlow, J.E. Settle, J.H. Fowler, A 61-million-person experiment in social influence and political mobilization, Nature 489 (7415) (2012) 295-298.

[5] F. Wu, B.A. Huberman, Novelty and collective attention, Proc. Natl. Acad. Sci. USA 104 (45) (2007) 17599-17601.

[6] L. Chen, Y. Zhou, D.M. Chiu, A lifetime model of online video popularity, in: 23rd International Conference on Computer Communication and Networks (ICCCN), IEEE, 2014, pp. 1-8.

[7] S. Fortunato, A. Flammini, F. Menczer, Scale-free network growth by ranking, Phys. Rev. Lett. 96 (21) (2006) 218701.

[8] A.H. Zadeh, R. Sharda, Modeling brand post popularity dynamics in online social networks, Decis. Support Syst. 65 (3) (2014) 59-68.

[9] L. Lü, M. Medo, C.H. Yeung, Y.C. Zhang, Z.K. Zhang, T. Zhou, Recommender systems, Phys. Rep. 519 (1)(2012) 1-49.

[10] Z. Huang, D.D. Zeng, H. Chen, Analyzing consumer-product graphs: Empirical findings and applications in recommender systems, Manag. Sci. 53 (7) (2007) 1146-1164.

[11] M.J. Salganik, P.S. Dodds, D.J. Watts, Experimental study of inequality and unpredictability in an artificial cultural market, Science 311 (5762) (2006) $854-856$.

[12] J.G. Liu, J.H. Lin, Q. Guo, T. Zhou, Locating influential nodes via dynamics-sensitive centrality, Sci. Rep. 6 (2016).

[13] Y.C. Zhang, M. Blattner, Y.K. Yu, Heat conduction process on community networks as a recommendation model, Phys. Rev. Lett. 99 (15) (2007) 154301.

[14] X.L. Liu, Q. Guo, L. Hou, C. Cheng, J.G. Liu, Ranking online quality and reputation via the user activity, Physica A 436 (2015) 629-636.

[15] Z.M. Ren, A. Zeng, D.B. Chen, H. Liao, J.G. Liu, Iterative resource allocation for ranking spreaders in complex networks, Europhys. Lett. 106 (4) (2014) 48005.

[16] A.L. Barabási, R. Albert, Emergence of scaling in random networks, Science 286 (5439) (1999) 509-512.

[17] G. Bianconi, A.L. Barabási, Competition and multiscaling in evolving networks, Europhys. Lett. 54 (4) (2001) 436.

[18] Y. Borghol, S. Ardon, N. Carlsson, D. Eager, A. Mahanti, The untold story of the clones: content-agnostic factors that impact YouTube video popularity, in: Proceedings of the 18th ACM SIGKDD International Conference on Knowledge Discovery and Data Mining, ACM, 2012 , pp. 1186-1194.

[19] G. Szabo, B.A. Huberman, Predicting the popularity of online content, Commun. ACM 53 (8) (2010) 80-88.

[20] H.W. Shen, D. Wang, C. Song, A.L. Barabási, Modeling and predicting popularity dynamics via reinforced Poisson processes. Proceedings of the 28th AAAI Conference on Artificial Intelligence Vol. 14, 2014, pp. 291.

[21] R.A. Bentley, P. Ormerod, M. Batty, Evolving social influence in large populations, Behav. Ecol. Sociobiol. 65 (3) (2011) $537-546$.

[22] J.P. Gleeson, D. Cellai, J.P. Onnela, M.A. Porter, F. Reed-Tsochas, A simple generative model of collective online behavior, Proc. Natl. Acad. Sci. USA 111 (29) (2014) 10411-10415.

[23] F.C. Santos, J.M. Pacheco, T. Lenaerts, Evolutionary dynamics of social dilemmas in structured heterogeneous populations, Proc. Natl. Acad. Sci. USA 103 (9) (2006) 3490-3494

[24] M. Medo, G. Cimini, S. Gualdi, Temporal effects in the growth of networks, Phys. Rev. Lett. 107 (23) (2011) 238701.

[25] J.G. Liu, T. Zhou, Q. Guo, Information filtering via biased heat conduction, Phys. Rev. E 84 (3) (2011) 037101.

[26] J. Ni, Y.L. Zhang, Z.L. Hu, W.J. Song, L. Hou, Q. Guo, J.G. Liu, Ceiling effect of online user interests for the movies, Physica A 402 (2014) $134-140$.

[27] J.H. Wang, Q. Guo, K. Yang, Y.L. Zhang, J.T. Han, J.G. Liu, Popularity and user diversity of online objects, Physica A 461 (2016) $480-486$.

[28] T. Zhou, J.G. Liu, W.J. Bai, G. Chen, B.H. Wang, Behaviors of susceptible-infected epidemics on scale-free networks with identical infectivity, Phys. Rev. E 74 (5) (2006) 056109.

[29] L. Ji, J.G. Liu, L. Hou, Q. Guo, Identifying the role of common interests in online user trust formation, PLoS One 10 (7) (2015) e0121105.

[30] J. Ratkiewicz, S. Fortunato, A. Flammini, F. Menczer, A. Vespignani, Characterizing and modeling the dynamics of online popularity, Phys. Rev. Lett. 105 (15) (2010) 158701

[31] J.P. Onnela, F. Reed-Tsochas, Spontaneous emergence of social influence in online systems, Proc. Natl. Acad. Sci. USA 107 (43) (2010) $18375-18380$.

[32] C. Liu, Z.K. Zhang, Information spreading on dynamic social networks, Commun. Nonlinear Sci. Numer. Simul. 19 (4) (2014) 896-904.

[33] L. Hou, X. Pan, Q. Guo, J.G. Liu, Memory effect of the online user preference, Sci. Rep. 4 (2014) 6560.

[34] K. Yang, Q. Guo, S.N. Li, J.T. Han, J.G. Liu, Evolution properties of the community members for dynamic networks, Phys. Lett. A 381 (11) (2017) 970-975. 
[35] Z.K. Zhang, C. Liu, X.X. Zhan, L. Xin, C.X. Zhang, Y.C. Zhang, Dynamics of information diffusion and its applications on complex networks, Phys. Rep. $651(2016) 1-34$

[36] Q.X. Wang, J.J. Zhang, X.Y. Shi, M.S. Shang, User Heterogeneity and Individualized Recommender, Chin. Phys. Lett. 34 (2017) 068902.

[37] Z.M. Ren, Y.Q. Shi, H. Liao, Characterizing popularity dynamics of online videos, Physica A 453 (2016) 236-241.

[38] L.Y. Tang, S.N. Li, J.H. Lin, Q. Guo, J.G. Liu, Community structure detection based on the neighbor node degree information, Internat. J. Modern Phys. C 27 (04) (2016) 1650046.

[39] M. Girvan, M.E.J. Newman, Community structure in social and biological networks, Proc. Natl. Acad. Sci. USA 99 (12) (2002) $7821-7826$.

[40] C.X. Zhang, Z.K. Zhang, L. Yu, C. Liu, H. Liu, X.Y. Yan, Information filtering via collaborative user clustering modeling, Physica A 396 (2013) $195-203$. 